\title{
Chronic disease and recent addiction treatment utilization among alcohol and drug dependent adults
}

Sharon Reif ${ }^{* *}$, Mary Jo Larson ${ }^{1}$, Debbie M Cheng ${ }^{2}$, Donald Allensworth-Davies ${ }^{2}$, Jeffrey Samet ${ }^{2,3,4}$ and Richard Saitz ${ }^{2,3,4}$

\begin{abstract}
Background: Chronic medical diseases require regular and longitudinal care and self-management for effective treatment. When chronic diseases include substance use disorders, care and treatment of both the medical and addiction disorders may affect access to care and the ability to focus on both conditions. The objective of this paper is to evaluate the association between the presence of chronic medical disease and recent addiction treatment utilization among adults with substance dependence.
\end{abstract}

Methods: Cross-sectional secondary data analysis of self-reported baseline data from alcohol and/or drugdependent adults enrolled in a randomized clinical trial of a disease management program for substance dependence in primary care. The main independent variable was chronic medical disease status, categorized using the Katz Comorbidity Score as none, single condition of lower severity, or higher severity (multiple conditions or single higher severity condition), based on comorbidity scores determined from self-report. Asthma was also examined in secondary analyses. The primary outcome was any self-reported addiction treatment utilization (excluding detoxification) in the 3 months prior to study entry, including receipt of any addiction-focused counseling or addiction medication from any healthcare provider. Logistic regression models were adjusted for sociodemographics, type of substance dependence, recruitment site, current smoking, and recent anxiety severity.

Results: Of 563 subjects, 184 (33\%) reported any chronic disease (20\% low severity; 13\% higher severity) and 111 (20\%) reported asthma; 157 (28\%) reported any addiction treatment utilization in the past 3 months. In multivariate regression analyses, no significant effect was detected for chronic disease on addiction treatment utilization (adjusted odds ratio [AOR] 0.88 lower severity vs. none, 95\% confidence interval (Cl): 0.60, 1.28; AOR 1.29 higher severity vs. none, $95 \% \mathrm{Cl}: 0.89,1.88)$ nor for asthma.

Conclusions: In this cohort of alcohol and drug dependent persons, there was no significant effect of chronic medical disease on recent addiction treatment utilization. Chronic disease may not hinder or facilitate connection to addiction treatment.

Keywords: addiction, substance abuse, substance abuse, treatment, medical care, chronic disease

\section{Introduction}

Chronic medical diseases are long-lasting conditions, often progressive, and often controllable with continuing care and behavior change. In an era of increasing health care costs [1], chronic disease stands out as a major

\footnotetext{
* Correspondence: reif@brandeis.edu

'Institute for Behavioral Health, Heller School for Social Policy and Management, Brandeis University, 415 South Street, MS035, Waltham, MA 02454, USA

Full list of author information is available at the end of the article
}

contributor [2] with alcohol and drug use disorders playing an exacerbating role [3-5]. To improve population health and reduce U.S. health care costs, particular attention must be paid to the role of chronic diseases, including medical, psychiatric, and substance use disorders.

Substance use disorders (SUDs) are prevalent in about $9 \%$ of the U.S. population, but only $10 \%$ of those individuals access addiction treatment [6]. Although medical needs were not explicitly mentioned in this household

\section{Biomed Central}


survey as a reason for not accessing addiction treatment [6], medical needs may interfere with other priorities. For example, chronic medical conditions could cause functional limitations that preclude access to other health care. Interference from pressing medical conditions may thus contribute to perceived unimportance of addiction treatment among the $94 \%$ of people [6] deemed in need but who reported they did not need it. To enhance care of both chronic medical diseases and addiction, it is important to better understand their relationships.

More than $40 \%$ of the U.S. population is estimated to have at least one chronic medical disease [2,7], with prevalence higher for women and increasing dramatically with age [7]. Chronic medical disease is related to $70 \%$ of all U.S. deaths [2], and accounts for more than $75 \%$ of health care costs [2] and high out of pocket health care spending [7]. Diabetes, hypertension, and high cholesterol comprise nearly a third of all chronic conditions [7], and just seven conditions account for about onefourth of annual outpatient visits and hospital discharges [8].

Chronic disease may have unexpected consequences on healthcare utilization, potentially reducing treatment of unrelated disorders, perhaps due to less time available, complexity of navigating multiple problems at once, or a preference to focus on the most troublesome problem [9]. In practice, however, perhaps due to more frequent interaction with medical providers, mental disorders are more often detected among patients with chronic medical diseases than among other patients [10]. Physician awareness of a substance abuse problem also is more likely when the person has episodic or chronic medical illness, however SUDs remain undiagnosed by primary care physicians in nearly half of patients seeking addiction treatment [11].

It is well documented that SUDs cause or exacerbate certain medical conditions and increase their costs [3,12-16]. Incidence for many chronic medical diseases, including hypertension, diabetes, asthma, chronic liver disease, chronic obstructive pulmonary disease (COPD), pain, and stroke is elevated, and for some disorders more than doubled, among people with SUDs [17-20]. Many more conditions are worsened by, have their management impaired by, or are attributable to SUDs [21]. Chronic conditions are prevalent even in younger individuals with SUDs [22]. These findings highlight the importance of medical management as an essential element of addiction treatment [23] particularly in early recovery [24].

Several examples of this complex interplay are reported in the literature. Alcohol use disorders increased medical illness complexity in a primary care population, even after controlling for medical morbidity, initial primary care utilization, and current medical care utilization [25]. Alcohol use disorders also were related to inconsistent attendance and less complex services for the chronic medical disease, suggesting that medical care may be less accessible for the comorbid population, who may require an increased focus on the complexities of care [26]. Among patients receiving diabetes care in the Department of Veterans Affairs, those who also had SUDs or mental illness had more diabetes complications [27]. Although SUDs have been linked to higher utilization of primary, specialty, and emergency medical services [25], there are few studies of how presence of complex medical disorders affects addiction treatment utilization.

Chronic medical diseases require regular and longitudinal care and self-management for effective treatment [28-30]. When chronic diseases include SUDs, care and treatment of both the medical and addiction disorders may affect access to care and the ability to focus on both conditions. An integrated approach could lead to effective treatment for all conditions [29], yet little is known about how chronic medical disease affects addiction treatment utilization. Chronic disease could cause functional limitations that interfere with an individual's ability to access addiction treatment, or alternatively could increase interaction with the health care system and perhaps addiction treatment. Involvement in addiction treatment could lead to linkage with medical care and effective attention to a chronic medical problem. The objective of this paper is to evaluate the association between the presence of chronic medical disease and recent addiction treatment utilization among adults with substance dependence.

\section{Methods}

\section{Data Source and Sample}

Data are from baseline interviews with 563 alcohol and/ or drug-dependent adults enrolled in the AHEAD (Addiction Health Evaluation And Disease management) study, a randomized controlled trial testing the effectiveness of a chronic disease management program for substance dependence in primary care.

Study recruitment occurred from a free-standing inpatient detoxification unit ( $74 \%$ of enrolled subjects), primary care clinics and the emergency department in the urban medical center where the study was located (10\%) and the community by advertising on buses and in newspapers (16\%); subjects were recruited between September 2006 and September 2008.

Adults were eligible if they had (1) a diagnosis of current alcohol or drug dependence as assessed by the Composite International Diagnostic Interview Short Form (CIDI-SF) [31]; (2) past 30 day drug use or heavy alcohol use (defined as $\geq 4$ standard drinks for women, 
$\geq 5$ for men at least twice; or $>14$ drinks per week for women, $>21$ drinks per week for men, in an average week in the past month); and (3) were willing to establish or continue primary medical care at the study location. Subjects were excluded if they could not provide contact information for tracking purposes; were not fluent in English or Spanish; had specific plans to leave the area that would preclude in-person research assessments; were pregnant; or had a Mini-Mental State Examination [32] score $<21$.

Trained research associates administered the standardized baseline research assessment and assured confidentiality. Data collected include sociodemographics, substance use and related problems, anxiety, depression, health questions, and medical and addiction service use. Subjects were compensated for their study participation after completing baseline study procedures.

The Institutional Review Board approved the study. Additional privacy protection was secured by a Certificate of Confidentiality issued by the Department of Health and Human Services.

\section{Chronic Disease and Other Medical Condition Measures}

Subjects were asked if a doctor had ever told them they had any of a series of medical conditions. A number of these conditions were included on a chronic disease comorbidity questionnaire validated by Katz et al. [33], which is a self-report version of the medical recordbased Charlson Comorbidity Index [34]. The Katz questionnaire includes myocardial infarction, congestive heart failure, peripheral vascular, stroke or other cerebrovascular disease, COPD, ulcer disease, diabetes, renal, connective tissue disease, and other conditions (dementia, liver disease, leukemia, lymphoma, cancer, AIDS). Scoring replicates the Charlson Comorbidity Index, which empirically weighted severity by disease (e. g., cerebrovascular $=1$, moderate or severe renal disease $=2$, AIDS or metastatic solid tumor $=6$ ), based on 1 year adjusted relative risk for mortality [34]. The comorbidity score is the weighted sum of the specified conditions.

The main independent variable, chronic disease status, is a 3-category variable which classified comorbidity scores as: none; only one condition of lowest severity $(\mathrm{Katz}=1)$; or, more than one condition of lowest severity or one or more condition(s) of higher severity (Katz $>1$ ). Asthma was considered in separate secondary analyses, as an example of a specific symptomatic condition, selected due to its high prevalence in the sample; it is also included in the Katz questionnaire, as part of COPD, with a weight of 1 . Asthma is a common comorbidity among urban young to middle-age adults, reflecting the age range common for addiction, whereas other common chronic diseases such as heart disease are less common in young populations. Two other common chronic conditions in this population, hepatitis and hypertension, were prevalent but are often asymptomatic to the patient, and thus were not evaluated in the current analyses.

Additional secondary independent variables were used to evaluate whether broader health status significantly predicted addiction treatment utilization: the single item self-report health status from the SF-12 [35] (excellent/ very good/good, fair/poor) and physical health-related functioning and quality of life as determined by the SF12 Physical Component Score (PCS) (continuous 0-100 scale).

\section{Addiction Treatment Utilization Measures}

The primary outcome was any addiction treatment based on self-report, defined as any treatment for addiction (excluding detoxification) in the 3 months prior to study enrollment in any of the following: residential program for alcohol or drug treatment; outpatient full-day/ partial hospital program; outpatient with a psychiatrist, other doctor, or other healthcare professional (e.g., counselor); or by taking medication to prevent drinking or drug use. Two secondary outcomes were the counts of outpatient addiction treatment days (at a full-day/partial hospital or with a psychiatrist, other doctor, or other healthcare professional including counselors) and nights in a residential program for alcohol or other drug treatment in the prior 3 months based on self-report at the baseline assessment. These measures were adapted from the COMBINE study Form 90 [36]. Self-report is known to be a reliable way of obtaining treatment utilization information among people with substance use disorders $[37,38]$.

\section{Covariates}

Demographic variables included age, gender, and race/ ethnicity. Current type of substance dependence is a 3category variable indicating either alcohol dependence with heavy alcohol use in the past 30 days, any drug dependence with drug use in the past 30 days, or both. Alcohol and drug dependence were determined using the CIDI-SF alcohol and drug modules [31]; heavy alcohol use in the past month was indicated by 4 or more drinks per day or 15 or more drinks per average week for women, and 5 or more drinks per day or 22 or more drinks per week for men. The regression models included other variables that may affect addiction treatment utilization: recruitment from a detoxification program (versus from medical care or the community), criminal justice involvement (any arrest, probation, parole, pretrial release, diversion program or incarceration in the past 3 months), homeless (any time living in a shelter or on the streets in the past 3 months), 
unemployed (usual pattern past 3 months), having health insurance (including Medicaid), current smoker, and anxiety in the past week (Beck Anxiety Index [39] scores: low $=0-21$, moderate $=22-35$, or high $=36$ or higher).

\section{Statistical Methods}

Secondary data analysis of the AHEAD study baseline data was conducted. Descriptive statistics were obtained and Spearman correlations were used to evaluate potential collinearity between independent variables and covariates. No pair of variables included in the same regression model was highly correlated $(r>0.40)$. Multivariate regression models were used to evaluate the effect of presence of chronic disease on addiction treatment utilization, adjusting for covariates defined above.

The primary outcome of any addiction treatment was modeled using logistic regression, with odds ratios and 95\% confidence intervals calculated. The distributions for outpatient addiction treatment days and residential nights, which are count variables, were skewed with many zeros and long tails, thus models assuming normality were inappropriate and overdispersed Poisson regression models were used [40]. The Pearson chisquare correction was used to account for overdispersion. The magnitudes of association between measures of chronic disease and utilization were quantified for the Poisson models using incidence rate ratios (IRRs) with 95\% confidence intervals. IRR is interpreted as the ratio of the number of outpatient days (or residential nights) for the exposed group of interest (e.g., any chronic disease) versus the reference group (no chronic disease). The null value of no association for the IRR is equal to 1. An IRR $>1$ indicates that chronic disease is associated with more treatment utilization.

Multivariate models were fit separately for each dependent variable (any addiction treatment, outpatient days, and residential nights) and for each key independent variable (chronic disease, asthma, health status, and PCS). The primary analyses using the Katz score included 498 subjects with complete data on outcomes, the main independent variable, and covariates; secondary models included the same 498 subjects. Power calculations assumed that $27 \%$ of subjects without chronic disease utilized addiction treatment (based on the observed data), giving our study approximately $80 \%$ power to detect an odds ratio as small as 1.9 for those with one chronic disease of lowest severity and an odds ratio as small as 2.1 for those with one chronic disease of higher severity or multiple chronic diseases. Thus, power was sufficient for effects that were clinically meaningful. All analyses were conducted using twosided tests and a significance level of 0.05, using SAS software (version 9.1; SAS Institute, Cary, NC).

\section{Results}

Baseline Characteristics

Of 2,018 potential subjects screened, 650 subjects were eligible, and 563 subjects were enrolled. Enrolled subjects were similar to unenrolled subjects in terms of gender, age and race/ethnicity. Baseline sociodemographic characteristics are noted in Table 1, as are prior healthcare and addiction treatment utilization.

Table 1 Baseline Characteristics $(n=563)$

\begin{tabular}{lcc}
\hline & $\mathbf{n}$ & $\%$ \\
\hline Age & Mean $=38.2$, range 18-67 \\
\hline Male & 409 & 73 \\
\hline Race & & \\
Black/African American & 185 & 33 \\
White & 272 & 48 \\
Other & 106 & 19 \\
\hline Hispanic or Latino & 76 & 14 \\
\hline Homeless (past 3 mo) & 332 & 59 \\
\hline Unemployed (past 3 mo) & 246 & 44 \\
\hline Has any insurance & 446 & 79 \\
\hline
\end{tabular}

Criminal justice involvement past 3 mo

$\begin{array}{lcc}\text { None } & 315 & 56 \\ \text { Probation } & 130 & 23 \\ \text { Pretrial release } & 63 & 11 \\ \text { Other/Missing } & 55 & 10\end{array}$

Substance dependence

Alcohol dependent \& recent heavy use $\quad 98 \quad 17$

$\begin{array}{lll}\text { Drug dependent } \& \text { recent use } & 150 & 27\end{array}$

Both

$315 \quad 56$

Substance reported as the major problem

Alcohol to intoxication

$142 \quad 25$

Heroin

$208 \quad 37$

Cocaine

81

Poly-substance or other drugs

132

14

Recruitment site

Detoxification unit

Ambulatory/outpatient/ER

Community/other

Current smoker

r

Beck anxiety score

Low $220 \quad 40$

Moderate 164

30

Severe

166

30

Prior healthcare utilization past 3 mo

Any emergency room

$304 \quad 54$

Any non-addiction outpatient

242

43

Addiction treatment utilization past 3 mo

Any (excluding detoxification)

157

Outpatient days

Residential nights 


\section{Addiction Treatment}

At baseline, $28 \%$ of subjects had been in addiction treatment in the prior 3 months, excluding detoxification (Table 1). The 97 patients (17\%) who had outpatient treatment in the prior 3 months attended a median of 5 days (interquartile range $3-13$ ). The 70 patients (12\%) with residential treatment in the past 3 months attended a median of 23 nights (interquartile range $8-45$ ).

\section{Chronic Disease, Physical Health and Other Medical Conditions}

Overall, two thirds of subjects had no chronic disease, $20 \%$ had one chronic disease of lower severity, and $14 \%$ had one of higher severity or multiple diseases (Table 2 ). One third of subjects reported their health status to be only poor or fair. The mean Physical Comorbidity Score is $41.7(\mathrm{SD}=8.4)$ (the general population norm is 50 [35]).

Subjects reported a wide range of medical conditions (Table 2). Hepatitis was reported by nearly one third of subjects, the most common single condition. Hypertension was reported by $21 \%$ of subjects and about $20 \%$ reported asthma. Ten to thirteen percent of subjects reported skin infections, pneumonia, anemia or seizures. Five to ten percent reported tachycardia, gastritis, diabetes, COPD, ulcer, or tuberculosis. Few (2-5\%) reported rheumatoid arthritis, neuropathy, heart conditions, stroke, HIV, pancreatitis, cancer, poor kidney function or cirrhosis, and remaining conditions were even more rare.

\section{Effect of Chronic Disease on Addiction Treatment Utilization}

In multivariate regression analyses (Table 3), no significant effect was detected for the main independent variable of chronic disease status on the odds of attending addiction treatment (adjusted odds ratio [AOR] 0.88 for lower severity vs. no chronic disease, 95\% confidence interval (CI): 0.60, 1.28; AOR 1.29 for higher severity vs. no chronic disease, $95 \%$ CI: $0.89,1.88)$. Similarly, no significant effect of chronic disease status was detected on number of outpatient days or residential nights.

For the primary outcome of any addiction treatment utilization (Model 1), being black was a negative factor, and having health insurance and severe anxiety were positively associated with any addiction treatment. Being female and having severe anxiety were positively associated with number of outpatient days, and recruitment from a detoxification facility was a negative factor (Model 2). Being black was a negative factor for residential nights (Model 3).

Similar results were found for the secondary independent variables of asthma and PCS, with neither significantly associated with any of the addiction
Table 2 Chronic Disease and Other Medical Conditions (n = 563)

\begin{tabular}{llc}
\hline & $\mathbf{n}$ & $\%$ \\
\hline Summary measures: & & \\
\hline Chronic disease (Katz) & 373 & 67 \\
$\quad$ None & 109 & 20 \\
$\quad$ One, of lower severity only & 75 & 14 \\
$\quad$ One of higher severity or multiple conditions & 48 & 9 \\
\hline Health status (self-reported) & 331 & 59 \\
$\quad$ Excellent & 184 & 33 \\
$\quad$ Very Good/Good & mean $=41.7$, range \\
$\quad$ Fair/Poor & \multicolumn{2}{c}{$17-60$}
\end{tabular}

Has a doctor ever told you that you had: ${ }^{1}$

\begin{tabular}{|c|c|c|}
\hline Hepatitis & 181 & 32 \\
\hline Hypertension* & 120 & 21 \\
\hline Asthma* & 111 & 20 \\
\hline Skin infections & 74 & 13 \\
\hline Pneumonia & 73 & 13 \\
\hline Anemia & 71 & 13 \\
\hline Seizures, epilepsy or convulsions & 60 & 11 \\
\hline Rapid heart beat or tachycardia & 45 & 8 \\
\hline Gastritis & 40 & 7 \\
\hline Diabetes* & 37 & 7 \\
\hline Emphysema, chronic bronchitis, COPD* & 35 & 6 \\
\hline Ulcer & 32 & 6 \\
\hline Tuberculosis & 28 & 5 \\
\hline Rheumatoid arthritis* & 24 & 4 \\
\hline Peripheral neuropathy & 21 & 4 \\
\hline Heart attack* & 19 & 3 \\
\hline Heart failure & 18 & 3 \\
\hline Stroke* & 16 & 3 \\
\hline $\mathrm{HIV}^{*}$ & 16 & 3 \\
\hline Pancreatitis & 14 & 2 \\
\hline Cancer* $^{*}$ & 13 & 2 \\
\hline Poor kidney function* & 12 & 2 \\
\hline Cirrhosis & 11 & 2 \\
\hline
\end{tabular}

* Condition included in Katz Comorbidity Index

$1<2 \%$ had blood clots in legs or lungs, septic arthritis, endocarditis, atrial fibrillation, cancer (mouth, throat, larynx, esophagus, stomach), peripheral vascular disease*, AIDS*, lupus*, leukemia* or polycythemia vera*, Alzheimer's or other dementia*, lymphoma*, kidney transplant* or polymylagia rheumatica*

treatment measures. Fair or poor health status was negatively associated with any addiction treatment and with outpatient addiction treatment, but not with residential treatment. Table 4 summarizes the results for each of the secondary independent variables (from separate regression models) on each of the outcome variables. 
Table 3 Multivariate Regression of Chronic Disease on Addiction Treatment Utilization $(\mathrm{N}=498)$

\begin{tabular}{|c|c|c|c|}
\hline & Model 1 & Model 2 & Model 3 \\
\hline & Any Addiction Treatment & Outpatient Days & Residential Nights \\
\hline Independent Variable & AOR $(95 \% \mathrm{Cl})$ & IRR (95\% CI) & IRR (95\% Cl) \\
\hline \multicolumn{4}{|l|}{ Chronic disease (Katz) } \\
\hline None & Referent & Referent & Referent \\
\hline One, of lower severity only & $0.88(0.60,1.28)$ & $0.86(0.41,1.81)$ & $0.68(0.31,1.49)$ \\
\hline One of higher severity or multiple conditions & $1.29(0.89,1.88)$ & $1.14(0.55,2.38)$ & $0.63(0.23,1.69)$ \\
\hline Age & $0.99(0.98,1.01)$ & $1.00(0.97,1.04)$ & $0.99(0.96,1.03)$ \\
\hline Female & $1.23(0.89,1.70)$ & $1.74(0.95,3.20)$ & $0.68(0.31,1.48)$ \\
\hline \multicolumn{4}{|l|}{ Race } \\
\hline Black & $0.57(0.38,0.85)^{* *}$ & $0.47(0.21,1.05)$ & $0.22(0.08,0.64)^{* *}$ \\
\hline Hispanic & $0.77(0.49,1.20)$ & $1.34(0.62,2.89)$ & $0.55(0.22,1.36)$ \\
\hline Other & $0.61(0.32,1.15)$ & $0.75(0.22,2.57)$ & $0.77(0.27,2.24)$ \\
\hline White & Referent & Referent & Referent \\
\hline \multicolumn{4}{|l|}{ Substance dependence } \\
\hline Alcohol dependent \& recent heavy use & $0.80(0.52,1.24)$ & $0.78(0.33,1.83)$ & $1.13(0.50,2.57)$ \\
\hline Drug dependent \& recent use & $0.86(0.61,1.22)$ & $0.87(0.39,1.95)$ & $0.48(0.21,1.11)$ \\
\hline Both & Referent & Referent & Referent \\
\hline \multicolumn{4}{|l|}{ Recruitment site } \\
\hline Detoxification program & $0.82(0.57,1.17)$ & $0.37(0.19,0.73)^{* *}$ & $1.11(0.48,2.57)$ \\
\hline Other & Referent & Referent & Referent \\
\hline Criminal justice involvement (past 3 mo) & $0.98(0.73,1.31)$ & $1.26(0.70,2.27)$ & $1.20(0.66,2.18)$ \\
\hline Homeless (past 3 mo) & $1.07(0.80,1.43)$ & $0.95(0.53,1.72)$ & $1.06(0.58,1.95)$ \\
\hline Unemployed (past 3 mo) & $1.07(0.79,1.45)$ & $1.60(0.79,3.24)$ & $0.85(0.47,1.54)$ \\
\hline Health insurance & $2.04(1.24,3.36)^{* *}$ & $3.06(0.88,10.68)$ & $2.54(0.96,6.71)$ \\
\hline Current smoker & $0.89(0.61,1.30)$ & $0.79(0.34,1.81)$ & $1.48(0.53,4.11)$ \\
\hline \multicolumn{4}{|l|}{ Anxiety (Beck) } \\
\hline Low & Referent & Referent & Referent \\
\hline Moderate & $1.45(0.99,2.14)$ & $1.71(0.80,3.66)$ & $2.15(0.97,4.75)$ \\
\hline Severe & $1.77(1.23,2.53)^{* *}$ & $2.12(1.02,4.41)^{*}$ & $2.11(0.94,4.75)$ \\
\hline
\end{tabular}

Model 1 used logistic regression; Models 2 and 3 used Poisson regression.

${ }^{*} \mathrm{p}<.05 * * \mathrm{p}<.01$; Wald chi-square tests, $\mathrm{df}=1$

\section{Discussion}

Despite the potential for interference or facilitation of chronic medical disease on utilization of addiction care, no statistically significant effect was found for chronic disease status on addiction treatment utilization in the prior 3 months, in this cohort of alcohol and drug dependent persons, controlling for sociodemographics, type of substance dependence, recruitment site, current

Table 4 Summary of Other Secondary Multivariate Regression Models, with alternate key independent variables ( $\mathrm{N}=$ 498)

\begin{tabular}{|c|c|c|c|}
\hline & Model 1 & Model 2 & Model 3 \\
\hline & Any Addiction Treatment & Outpatient Days & Residential Nights \\
\hline Key Independent Variable & AOR $(95 \% \mathrm{CI})$ & IRR $(95 \% \mathrm{Cl})$ & IRR (95\% Cl) \\
\hline Asthma & $1.05(0.74,1.49)$ & $1.03(0.52,2.03)$ & $1.03(0.50,2.12)$ \\
\hline \multicolumn{4}{|l|}{ Self-reported health status } \\
\hline Excellent/Nery Good/Good & Referent & Referent & Referent \\
\hline Fair/Poor & $0.67(0.48,0.94)^{*}$ & $0.40(0.20,0.80)^{* *}$ & $0.61(0.31,1.21)$ \\
\hline Physical Component Score (per 1 point increase) & $1.01(0.99,1.03)$ & $1.00(0.97,1.04)$ & $1.01(0.97,1.04)$ \\
\hline
\end{tabular}

Model 1 used logistic regression; Models 2 and 3 used Poisson regression. Models duplicate those in Table 3 (controlling for age, gender, race, substance dependence, recruitment from detox, criminal justice involvement, homeless, unemployed, health insurance, smoker, anxiety), solely replacing the chronic disease variable with the secondary variables above.

${ }^{*} \mathrm{p}<.05{ }^{* *} \mathrm{p}<.01 ;$ Wald chi-square tests, $\mathrm{df}=1$ 
smoking, and severity of recent anxiety. Nor was addiction treatment associated with asthma or physical function and quality of life. These findings held for any addiction treatment and for quantity of treatment services received. Only perceived health status, which is a subjective measure, was associated with less addiction treatment utilization. It is encouraging to note that chronic disease itself may not be a barrier to addiction treatment.

On the other hand, it also appears that chronic medical disease did not increase addiction treatment participation in this cohort. People with chronic disease are often connected to the medical treatment system, but the acute focus of episodic care may not extend to providing assistance or motivation for these patients to access addiction treatment. This may be a missed opportunity for medical providers to encourage their patients to seek addiction treatment given that the selfreported perception of health status was frequently only fair or poor. The lack of a significant positive association between chronic disease and addiction treatment utilization suggests that facilitated access to addiction care is not routinely occurring. Such "reachable moments" [41] for the individual with both chronic disease and addiction are perhaps most likely when formalized linkages between primary care and addiction treatment exist or if the primary care doctor is willing to address addiction with the patient $[18,42]$. One way to increase these reachable moments is to encourage screening, brief intervention, and referral to treatment approaches in the primary care setting. Opportunities for referral to addiction treatment should increase as the number of medical visits increases. An integrated, longitudinal approach to managing both chronic medical disease and substance dependence is likely to be most beneficial $[13,43,44]$.

It is worthwhile to note that no chronic diseases were reported by two thirds of this sample of substance dependent individuals, somewhat better than an estimate of $53 \%$ with no chronic conditions in a comparable population 10 years earlier [22]. The lower estimate here may be due to recruitment beyond detoxification settings; subjects recruited from the community may be less medically ill than those in detoxification facilities. Most of the chronic diseases noted here had prevalence within the range of national and state estimates $[45,46]$. The asthma prevalence here appears slightly higher than the state average, but this likely reflects the higher rates often found in inner city and African-American populations [47] and the high prevalence of current smoking in this sample. The below average Physical Comorbidity Score in this population is comparable to similar populations $10-15$ years earlier $[22,48]$.

Although we did not detect an association between chronic medical variables and treatment utilization, several other variables were significantly related to treatment utilization, including several sociodemographic variables. Health insurance was positively associated with any addiction treatment utilization, demonstrating an expected increase in access to treatment, but did not reach significance for the count variables. Recruitment from detoxification was negatively associated with outpatient days, highlighting the severity of addiction treatment needs for this group, and the expected lack of treatment preceding detoxification entry. Psychiatric comorbidity (e.g., anxiety) was positively associated with addiction treatment utilization reflecting other findings in the literature [49], although this past-week measure was collected for most individuals soon after detoxification, so may reflect short-term anxiety symptoms rather than a chronic disorder.

The lack of findings concerning our primary hypothesis may simply be the reflection of a complex relationship between chronic disease and addiction, with many competing forces. Several opposing effects from chronic disease may have occurred, which could collectively show little or no effect on addiction treatment utilization. First, individuals with chronic disease may be functionally less able to access and participate in addiction treatment, reducing the likelihood of treatment utilization. If physical function were an important barrier, we should have seen some effect from the PCS, which did not occur, but it might be important to consider specific functional limitations. By considering asthma separately, thus removing the variability across conditions, we might have highlighted the functional effects, but that also did not occur. It is also possible that the severity of addiction, as indicated by most individuals in the sample recruited from detoxification, overshadowed any functional limitations as they sought treatment; a desire to focus on the most pressing problem has been highlighted in some literature [9]. Second, for a variety of reasons, addiction treatment facilities may be less likely to accept individuals with medical conditions, who may require medications or have more acute medical needs. This study could not address that question. Third, chronic disease may interfere with the quality of treatment participation; number of visits could be construed as a proxy for this, but if chronic disease affects selfcare management this issue may go beyond what could be considered here. Fourth, 3 months is a relatively short time period, so may have masked any differences that would appear with longer history of treatment utilization. However, the focus here is on relative differences in utilization, so the 3 month period should have been sufficient to compare groups. Fifth, and alternatively, individuals with chronic disease may be more likely to access treatment due to their likely existing linkages with the healthcare system. A study of women with 
trauma and mental or substance use disorders found that greater disability was associated with more outpatient counseling, but not residential, group counseling or peer treatment [50]. While referral from the healthcare system is not a common pathway to addiction treatment [51] the finding that physicians are more likely to know about substance abuse if the patient has an episodic or chronic medical condition [11] suggests that this pathway may be more likely for these patients.

The characteristics of the study sample may have tempered the potential relationships between chronic disease and addiction treatment utilization. In a relatively young sample such as this, chronic disease is less likely, and related problems are likely to be less severe than in an older population. However, addiction itself may increase such problems even in a young sample [22]. The subjects in this study are largely reflective of individuals in addiction treatment, and these results should apply to similar cohorts. Relatively few individuals in this sample had severe chronic disease or multiple conditions, thus findings may be different in an analysis of only individuals with chronic disease. The implications of recruiting much of the sample during a detoxification stay should be considered, where individuals entering detoxification may be unlikely to have been in addiction treatment during a period of substance use severe enough to warrant detoxification. These findings also represent a sample from a single site, so may be less generalizable than recruitment from multiple sites.

A limitation is that the study may have been inadequately powered to detect differences of the observed magnitude. Power calculations estimated $80 \%$ power to detect an odds ratio as small as 2.1 for those with higher severity chronic disease, thus it is likely that the study was not adequately powered to detect the small observed magnitude of association of 1.3 (for higher severity). However, since the observed degree of association may still be clinically meaningful, the possibility that individuals with higher comorbidity are more likely to utilize addiction treatment should be further studied in a larger sample to see if a small but clinically important effect is detectable. But the effect of greater concern, interference with receiving addiction treatment due to a chronic disease, is unlikely based on our findings. Last, the current analysis relied on retrospective data from a sample of individuals, most of whom had recently completed detoxification. A prospective or qualitative study would be better able to track the likely causal issues related to chronic disease and addiction treatment access.

To our knowledge, this is the first analysis of the role of chronic disease in addiction treatment utilization. Current household studies on barriers to addiction treatment, such as SAMHSA's National Survey on Drug
Use and Health (NSDUH), do not consider medical comorbidities, so are unable to shed light on these underlying questions. Further research in this area is warranted to better understand these complex issues. The individuals in this study were, by definition, willing to receive health care. Other populations may differ. Also, samples with a large number of individuals with chronic disease would allow a focus on more specific chronic diseases, such as diabetes or heart disease, and would allow a better consideration of other psychiatric comorbidity. In the meantime, we highlight the conclusion from these data that chronic disease is not a significant barrier to addiction treatment utilization. While we found no significant association between chronic medical disease and addiction treatment utilization, these issues are complicated and we suggest prospective and qualitative studies to further explore these complexities.

\section{Acknowledgements}

Preliminary results of this study were presented at the Integrating Services \& Research for Co-Occurring Conditions conference, Bethesda, MD, March 2009, and the Association for Medical Education and Research in Substance Abuse conference, Bethesda MD, November 2009. This study was supported by the National Institute on Alcohol Abuse and Alcoholism (grant R01 AA010870) and the National Institute on Drug Abuse (grant R01 DA010019). This work was also supported, in part, by the Boston University General Clinical Research Unit which is part of the Clinical and Translational Science Institute (National Center for Research Resources (NCRR) Grant Number UL1RR025771), and the Brandeis/Harvard NIDA Research Center (P50 DA010233). JS receives support from NIAAA K24 AA015674. The authors thank Julie Witas for project management, and the AHEAD study research team and subjects.

\section{Author details}

${ }^{1}$ Institute for Behavioral Health, Heller School for Social Policy and Management, Brandeis University, 415 South Street, MS035, Waltham, MA 02454, USA. ${ }^{2}$ Boston University School of Public Health, 801 Massachusetts Avenue, Boston, MA 02118, USA. ${ }^{3}$ Boston University School of Medicine, 801 Massachusetts Avenue, Boston, MA 02118, USA. ${ }^{4}$ Boston Medical Center, 801 Massachusetts Avenue, Boston, MA 02118, USA.

\section{Authors' contributions}

All authors contributed to the design, interpretation of results, and the manuscript preparation and have approved the final manuscript. In addition, significant individual contributions are as follows. SR conceived of the study, designed the analytic plan, and led the writing, interpretation of results, and revisions of the manuscript. MJL contributed to the study scope, analytic plan, and interpretation of results. DMC provided statistical expertise, and contributed to the study scope, analytic plan, and interpretation of results. DA conducted the statistical analysis. RS and JS lead the parent study from which the data were obtained, brought expertise regarding the data and the study population, and contributed to the study scope, analytic plan, and interpretation of results.

\section{Competing interests}

The authors declare that they have no competing interests.

Received: 9 June 2011 Accepted: 18 October 2011

Published: 18 October 2011

\section{References}

1. National Health Expenditure Data, Historical. [http://www.cms.hhs.gov/ NationalHealthExpendData/02_NationalHealthAccountsHistorical. asp\#TopOfPage]. 
2. Chronic Disease Overview. [http://www.cdc.gov/NCCdphp/overview.htm]

3. Clark RE, Samnaliev M, McGovern MP: Impact of substance disorders on medical expenditures for medicaid beneficiaries with behavioral health disorders. Psychiatr Senv 2009, 60:35-42.

4. Mark TL, Levit KR, Coffey RM, McKusick DR, Harwood HJ, King EC, Bouchery E, Genuardi JS, Vandivort-Warren R, Buck JA, Ryan K: National Expenditures for Mental Health Services and Substance Abuse Treatment, 1993-2003. Rockville, MD: Substance Abuse and Mental Health Services Administration; 2007.

5. Office of National Drug Control Policy: The Economic Costs of Drug Abuse in the United States, 1992-2002. Washington, D.C.: Executive Office of the President; 2004.

6. Substance Abuse and Mental Health Services Administration: Results from the 2008 National Survey on Drug Use and Health: National Findings. Rockville, MD: Office of Applied Studies; 2009.

7. Paez KA, Zhao L, Hwang W: Rising out-of-pocket spending for chronic conditions: a ten-year trend. Health Aff (Millwood) 2009, 28:15-25.

8. Decker SL, Schappert SM, Sisk JE: Use of medical care for chronic conditions. Health Aff (Millwood) 2009, 28:26-35.

9. Redelmeier DA, Tan SH, Booth GL: The treatment of unrelated disorders in patients with chronic medical diseases. N Engl J Med 1998, 338:1516-1520.

10. Borowsky SJ, Rubenstein LV, Meredith LS, Camp P, Jackson-Triche M, Wells KB: Who is at risk of nondetection of mental health problems in primary care? J Gen Intern Med 2000, 15:381-388.

11. Saitz R, Mulvey KP, Plough A, Samet JH: Physician unawareness of serious substance abuse. Am J Drug Alcohol Abuse 1997, 23:343-354.

12. Graham AW, Schultz TK, Mayo-Smith MF, Ries RK, Wilford BB: Principles of Addiction Medicine. 3 edition. Chevy Chase, MD: American Society of Addiction Medicine; 2003.

13. Institute of Medicine: Improving the Quality of Health Care for Mental and Substance-Use Conditions Washington, D.C.: National Academies Press; 2006.

14. Banerjea R, Sambamoorthi U, Smelson D, Pogach LM: Expenditures in mental illness and substance use disorders among veteran clinic users with diabetes. J Behav Health Serv Res 2008, 35:290-303.

15. Garnick DW, Hendricks AM, Comstock C, Horgan C: Do individuals with substance abuse diagnoses incur higher charges than individuals with other chronic conditions? J Subst Abuse Treat 1997, 14:457-465.

16. Thomas MR, Waxmonsky JA, Gabow PA, Flanders-McGinnis G, Socherman R, Rost K: Prevalence of psychiatric disorders and costs of care among adult enrollees in a Medicaid HMO. Psychiatr Serv 2005, 56:1394-1401.

17. Wadland WC, Ferenchick GS: Medical comorbidity in addictive disorders. Psychiatr Clin North Am 2004, 27:675-687.

18. Mertens JR, Lu YW, Parthasarathy S, Moore C, Weisner CM: Medical and psychiatric conditions of alcohol and drug treatment patients in an HMO: comparison with matched controls. Arch Intern Med 2003, 163:2511-2517.

19. Larson MJ, Paasche-Orlow M, Cheng DM, Lloyd-Travaglini C, Saitz R, Samet JH: Persistent pain is associated with substance use after detoxification: a prospective cohort analysis. Addiction 2007, 102:752-760.

20. Dickey B, Normand SL, Weiss RD, Drake RE, Azeni H: Medical morbidity, mental illness, and substance use disorders. Psychiatr Serv 2002, 53:861-867.

21. Fox K, Merrill JC, Chang HH, Califano JA Jr: Estimating the costs of substance abuse to the Medicaid hospital care program. Am J Public Health 1995, 85:48-54.

22. De Alba I, Samet JH, Saitz R: Burden of medical illness in drug- and alcohol-dependent persons without primary care. Am J Addict 2004 13:33-45.

23. Mee-Lee D, Shulman GD, Fishman M, Gastfriend DR, Griffith JH, (Eds.): ASAM Patient Placement Criteria for the Treatment of Substance-Related Disorders, 2nd edition-revised Chevy Chase, MD: American Society of Addiction Medicine; 2001.

24. Schulz JE: The integration of medical management with recovery. J Psychoactive Drugs 1997, 29:233-237.

25. Ford JD, Trestman RL, Steinberg K, Tennen H, Allen S: Prospective association of anxiety, depressive, and addictive disorders with high utilization of primary, specialty and emergency medical care. Soc Sci Med 2004, 58:2145-2148.

26. Ford JD, Trestman RL, Tennen H, Allen S: Relationship of anxiety, depression and alcohol use disorders to persistent high utilization and potentially problematic under-utilization of primary medical care. Soc Sci Med 2005, 61:1618-1625.

27. Banerjea R, Sambamoorthi U, Smelson D, Pogach LM: Chronic illness with complexities: mental illness and substance use among Veteran clinic users with diabetes. Am J Drug Alcohol Abuse 2007, 33:807-821.

28. Merrick EL, Horgan CM, Garnick DW, Hodgkin D, Morley M: Health plans' disease management programs: extending across the medical and behavioral health spectrum? J Ambul Care Manage 2008, 31:342-353.

29. Saitz R, Larson MJ, LaBelle C, Richardson J, Samet JH: The case for chronic disease management for addiction. J Addict Med 2008, 2:55-65.

30. Wagner EH, Austin BT, Von Korff M: Organizing care for patients with chronic illness. Milbank Q 1996, 74:511-544.

31. Kessler RC, Andrews G, Mroczek D, Ustun B, Wittchen HU: The World Health Organization Composite International Diagnostic Interview shortform (CIDI-SF). Int J Methods Psychiatr Res 1998, 7:171-185.

32. Smith KL, Horton NJ, Saitz R, Samet JH: The use of the mini-mental state examination in recruitment for substance abuse research studies. Drug Alcohol Depend 2006, 82:231-237.

33. Katz JN, Chang LC, Sangha O, Fossel AH, Bates DW: Can comorbidity be measured by questionnaire rather than medical record review? Med Care 1996, 34:73-84

34. Charlson ME, Pompei P, Ales KL, Mackenzie CR: A new method of classifying prognostic comorbidity in longitudinal studies: development and validation. J Chronic Dis 1987, 40:373-383.

35. Ware J Jr, Kosinski M, Keller SD: A 12-Item Short-Form Health Survey: construction of scales and preliminary tests of reliability and validity. Med Care 1996, 34:220-233.

36. Anton RF, O'Malley SS, Ciraulo DA, Cisler RA, Couper D, Donovan DM, Gastfriend DR, Hosking JD, Johnson BA, LoCastro JS, et al: Combined pharmacotherapies and behavioral interventions for alcohol dependence: the COMBINE study: a randomized controlled trial. JAMA 2006, 295:2003-2017.

37. Beebe TJ, McRae JA Jr, Barnes SA: A comparison of self-reported use of behavioral health services with Medicaid agency records in Minnesota. Psychiatr Serv 2006, 57:1652-1654.

38. Killeen TK, Brady KT, Gold PB, Tyson C, Simpson KN: Comparison of selfreport versus agency records of service utilization in a community sample of individuals with alcohol use disorders. Drug Alcohol Depend 2004, 73:141-147.

39. Beck AT, Epstein N, Brown G, Steer RA: An inventory for measuring clinical anxiety: psychometric properties. J Consult Clin Psychol 1988, 56:893-897.

40. McCullagh P, Nelder JA: Generalized Linear Models. 2 edition. London: Chapman and Hall; 1989.

41. Shanahan CW, Beers D, Alford DP, Brigandi E, Samet JH: A transitional opioid program to engage hospitalized drug users. $J$ Gen Intern Med 2010, 25:803-808.

42. Samet JH, Friedmann P, Saitz R: Benefits of linking primary medical care and substance abuse services: patient, provider, and societal perspectives. Arch Intern Med 2001, 161:85-91.

43. Weisner C, Mertens J, Parthasarathy S, Moore C, Lu Y: Integrating primary medical care with addiction treatment: a randomized controlled trial. JAMA 2001, 286:1715-1723.

44. Weisner C, Ray GT, Mertens JR, Satre DD, Moore C: Short-term alcohol and drug treatment outcomes predict long-term outcome. Drug Alcohol Depend 2003, 71:281-294.

45. National Center for Health Statistics: Summary Health Statistics for U.S. Adults: National Health Interview Survey, 2008 (provisional report). Vital and Health Statistics, Series 10, Number 242 Hyattsville, MD: Centers for Disease Control and Prevention; 2009

46. Massachusetts Department of Public Health: A Profile of Health Among Massachusetts Adults, 2008: Results from the Behavioral Risk Factor Surveillance System. Boston, MA: Massachusetts Department of Public Health; 2009.

47. Gold DR, Wright R: Population disparities in asthma. Annu Rev Public Health 2005, 26:89-113.

48. Stein MD, Mulvey KP, Plough A, Samet JH: The functioning and well being of persons who seek treatment for drug and alcohol use. J Subst Abuse 1998, 10:75-84.

49. Ilgen MA, Price AM, Burnett-Zeigler I, Perron B, Islam K, Bohnert AS, Zivin K: Longitudinal predictors of addictions treatment utilization in treatment- 
naive adults with alcohol use disorders. Drug Alcohol Depend 2011,

113:215-221.

50. Larson MJ, Miller L, Becker M, Richardson E, Kammerer N, Thom J, Gampel J, Savage A: Physical health burdens of women with trauma histories and co-occurring substance abuse and mental disorders. J Behav Health Serv Res 2005, 32:128-140

51. Substance Abuse and Mental Health Services Administration: Treatment Episode Data Set (TEDS) - Highlights 2007. National Admissions to Substance Abuse Treatment Services. Rockville, MD: Office of Applied Studies; 2009.

doi:10.1186/1747-597X-6-28

Cite this article as: Reif et al:: Chronic disease and recent addiction treatment utilization among alcohol and drug dependent adults.

Substance Abuse Treatment, Prevention, and Policy 2011 6:28.

Submit your next manuscript to BioMed Central and take full advantage of:

- Convenient online submission

- Thorough peer review

- No space constraints or color figure charges

- Immediate publication on acceptance

- Inclusion in PubMed, CAS, Scopus and Google Scholar

- Research which is freely available for redistribution

Submit your manuscript at www.biomedcentral.com/submit 